\title{
Design of PI Controllers for Hydraulic Control Systems
}

\author{
LJubiša Dubonjić, Novak Nedić, Vojislav Filipović, and Dragan Pršić \\ Faculty of Mechanical Engineering Kraljevo, University of Kragujevac, Dositejeva 19, 36000 Kraljevo, Serbia \\ Correspondence should be addressed to LJubiša Dubonjić; dubonjic.lj@mfkv.kg.ac.rs
}

Received 7 December 2012; Accepted 14 February 2013

Academic Editor: Zoran Gajic

Copyright (C) 2013 LJubiša Dubonjić et al. This is an open access article distributed under the Creative Commons Attribution License, which permits unrestricted use, distribution, and reproduction in any medium, provided the original work is properly cited.

\begin{abstract}
The paper proposes a procedure for design of PI controllers for hydraulic systems with long transmission lines which are described by models of high order. Design is based on the combination of the IE criterion and engineering specifications (settling time and relative stability) as well as on the application of $D$-decomposition. In comparison with some known results, the method is of graphical character, and it is very simple (solving nonlinear algebraic equations is eliminated). The paper presents the algorithm of software procedure for design of the controller. The method is compared with other methods at the level of simulation, and its superiority is shown. By applying the Nyquist criterion, it is shown that the method possesses robustness in relation to non modelled dynamics.
\end{abstract}

\section{Introduction}

The first heuristic rules for selection of parameters of the PI controller were given by Ziegler and Nichols in 1942 [1]. Since then, numerous papers have been devoted to determination of parameters of the controller, according to different types of assumed controlled processes. It is estimated that more than $95 \%$ of controllers used in industry are PID controllers, and most of them are PI controllers [2]. The performed analysis connected with the efficiency of control contours in industrial production has shown that PI controllers are used to a large extent, but they are commonly tuned in a bad manner [3, 4]. According to [4], only $20 \%$ of controllers in industry operate well, while $30 \%$ of them have poor performance due to the incorrectly tuned controller. In [5], based on research in process industry, the managers and engineers reach a common conclusion that tuning of parameters of PI and PID controllers is an important and difficult problem that deserves more attention. According to [6], the PI controller is the most frequently used control algorithm in process industry. The reason for that is its relatively simple structure, which can be easily understood and implemented in practice. Despite its wide application, there is still a need for improved tuning of parameters of the PI controller [7]. The heuristic method proposed by Ziegler and Nichols has the great advantage because it requires very little information about the process. However, this method has significant disadvantages, and it gives very bad damping (it is usually $\xi=0.2$ ) [7]. Small damping leads to degradation of the system and very bad performances from the aspect of relative stability and robustness.

Most PI controllers in process industry operate in the regime of set point value. Therefore, it is of principal importance to efficiently solve the problem of load disturbance rejection [8]. Reference [9] considers optimal tuning of controllers. It is shown that the IE criterion (integrated error) is in direct relation with integral gain of controllers. Those results are further expanded in [10], where it is proposed to have optimal load disturbance rejection with constraints on the function of system sensitivity and the weighting coefficient of the given value. The problem is reduced to solv nonlinear algebraic equations. The Newton-Raphson method is used for solving these equations. The initial conditions are the critical factor in this method.

Reference [11] presents a simple procedure for tuning of the PI controller. The problem is reduced to minimization of the IE criterion with the constraint that the Nyquist curve of the loop transfer function has a tangent in its left semiplane, parallel to an imaginary axis. The result of the procedure is explicit formulas for proportional and integral gains. Reference [12] proposes a new procedure for tuning of ideal PID controllers in series with the first-order noise filter. 
The problem is reduced to solving two nonlinear algebraic equations.

Reference [13] gives the procedure for synthesis of the $\mathrm{P}$ controller based on the generalized Nyquist criterion for hydraulic systems with long transmission lines described by models of high order. Such a type of controllers cannot eliminate the static error of control. That weakness is eliminated by the PI controller. The method that enables design of the PI controller for systems of high order is $D$ decomposition. The $D$-decomposition method was developed by Neimark $[14,15]$. It was considerably expanded by Mitrović establishing a strong link between the values of tuning parameters of the characteristic polynomial and the appearance of the transition process expressed through the corresponding degree of relative stability of the system [16]. The $D$-decomposition method was fully generalized in the algebraic method developed by Siljak in [17-19]. For efficient interpretation of results obtained by the $D$-decomposition method it is also necessary to have appropriate graphical interpretation which, for the systems of high order, was not possible without the corresponding software support. Intensive development of computer technique has renewed the interest in this methodology [20]. Let us emphasize that modern control theory allows design of controllers, for processes of high order, whose order will be equal to the order of the process [21-24]. Implementation of such controllers in industry is very complex and expensive. From that aspect, there is a need for design of controllers of low order, which are most frequently present in industrial practice, for control of processes of high order [25-27].

This paper proposes the procedure for design of the PI controller for systems of high order. The results from [9], which establishes a direct relation between the IE criterion and the integral gain (the higher the integral gain, the smaller the value of the IE criterion), are the starting point. The result is extended by introducing engineering specifications (settling time and relative stability). It results in a simple and efficient procedure for design of the PI controller for systems of high order. The method is basically graphical, and unlike $[10,12]$, it is not necessary to solve any nonlinear algebraic equations. The complete methodology of design is given in the second chapter of this paper. The third chapter gives the results of simulation for the hydraulic system with long transmission lines. A MATLAB program was created for graphical interpretation of results, and its flow is presented by the algorithm given in this chapter. The fourth chapter presents the results of comparative analysis of the proposed method for design of the PI controller and other methods, showing the superiority of the method proposed in this paper.

\section{Design of the PI Controller for Systems of High Order}

In [9], the problem of disturbance rejection is reduced to minimization of the following IE criterion:

$$
\mathrm{IE}=\int_{0}^{\infty} e(t) d t
$$

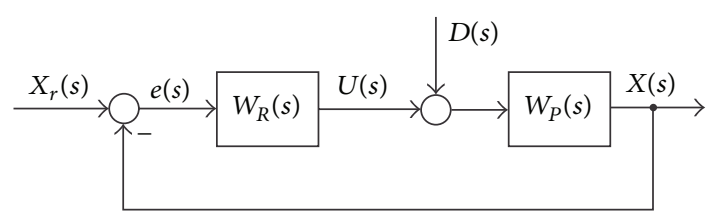

FIGURE 1: Automatic control system.

As the result, the following relation is obtained:

$$
\mathrm{IE}=\frac{1}{K_{i}}
$$

The result is extended in [10] by introducing constraints on the sensitivity function and the weighting factor of the set point value in the optimization problem. The problem belongs to the category of nonconvex optimization and is reduced to solving nonlinear algebraic equations by iterative methods.

This paper also considers the IE criterion, but instead of constraints from [10], engineering constraints are introduced on

(i) relative stability (damping of the closed loop) and

(ii) settling time.

The solution of the problem lies in the intersection between the previous two conditions. In accordance with the minimum of the IE criterion, the additional condition is that the integral gain should have the maximum value. This is the basis for development of a simple graphical method based on D-decomposition.

The transfer function of the PI controller is

$$
W_{R}=K_{p}+\frac{K_{i}}{s}
$$
form:

The transfer function of the process is represented in the

$$
W_{P}(s)=\frac{N(s)}{M(s)}=\frac{\sum_{k=0}^{m} b_{k} s^{k}}{\sum_{k=0}^{n} a_{k} s^{k}}, \quad m \leq n .
$$

The characteristic equation of the automatic control system from Figure 1 is determined by the equation:

$$
\begin{aligned}
& f(s)=1+W_{R}(s) W_{P}(s)=0, \\
& f(s)=1+\left(K_{p}+\frac{K_{i}}{s}\right) \cdot \frac{N(s)}{M(s)}=0, \\
& f(s)=s \cdot M(s)+\left(K_{p} s+K_{i}\right) \cdot N(s)=0, \\
& f_{1}(s)=s \cdot M(s)=\sum_{k=0}^{n} a_{k} s^{k+1} .
\end{aligned}
$$

By connecting (7) and (8), the final expression for the characteristic equation of the automatic control system in the complex domain is obtained as follows:

$$
f(s)=f_{1}(s)+\left(K_{p} s+K_{i}\right) \cdot N(s)=0 .
$$




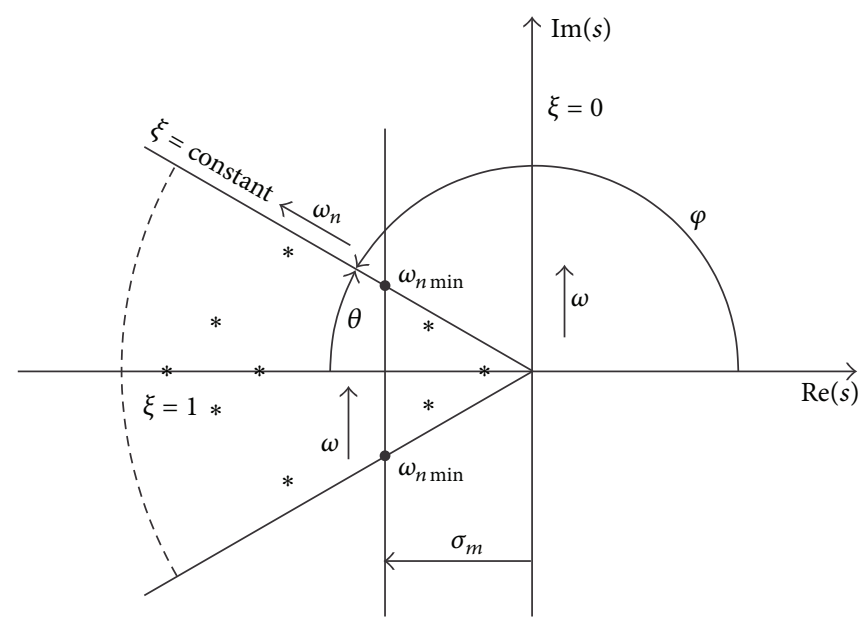

FIGURE 2: Area with the required settling time and relative stability.

Taking into account (9), it is necessary to express the complex number $s$ in a suitable form and use it for establishing the relation between the damping degree $\xi$ and the variable parameters of the controller, $K_{p}$ and $K_{i}$, contained in the characteristic equation (9) for the automatic control system. This is how the area from the " $s$ " plane below the straight line $\xi=$ const. (Figure 2) is mapped in the area of the corresponding damping coefficient represented by the curve $\xi=$ const., in the parameter plane of tuning parameters of the controller $\left(K_{p}, K_{i}\right)$ as follows:

$$
s=\omega_{n} e^{j \phi}=\omega_{n} e^{j(\pi-\theta)}=-\omega_{n} e^{-j \theta}=-\omega_{n} \cos \theta+j \omega_{n} \sin \theta,
$$

where

$$
\begin{aligned}
& \xi=\cos \theta, \quad 0 \leq \xi \leq 1, \\
& s=-\omega_{n} \xi+j \omega_{n} \sqrt{1-\xi^{2}} .
\end{aligned}
$$

By connecting (9) with (12), the characteristic equation of the automatic control system obtains the form:

$$
\begin{gathered}
f_{1}\left(\xi, \omega_{n}\right)+\left[K_{p}\left(-\xi \omega_{n}+j \omega_{n} \sqrt{1-\xi^{2}}\right)+K_{i}\right] \\
\cdot N\left(\xi, \omega_{n}\right)=0 \\
f_{1}\left(\xi, \omega_{n}\right)=\alpha\left(\xi, \omega_{n}\right)+j \beta\left(\xi, \omega_{n}\right),
\end{gathered}
$$

where $\alpha\left(\xi, \omega_{n}\right)$ and $\beta\left(\xi, \omega_{n}\right)$ represent the real and imaginary parts of the polynomial $f_{1}\left(\xi, \omega_{n}\right)$. The real and imaginary parts of the polynomial $f_{1}\left(\xi, \omega_{n}\right)$ are obtained by connecting (8) with (12) as follows:

$$
\begin{aligned}
& \alpha\left(\xi, \omega_{n}\right)=\sum_{k=1}^{n} a_{k-1}(-1)^{k} \omega_{n}^{k} T_{k}(\xi), \\
& \beta\left(\xi, \omega_{n}\right)=\sqrt{1-\xi^{2}} \sum_{k=1}^{n} a_{k-1}(-1)^{k+1} \omega_{n}^{k} U_{k}(\xi),
\end{aligned}
$$

where $T_{k}$ and $U_{k}$ are Chebyshev functions of the first and second kinds for which the following recurrent equations hold:

$$
\begin{gathered}
T_{k+1}=2 \xi T_{k}-T_{k-1}, \quad U_{k+1}=2 \xi U_{k}-U_{k-1}, \\
T_{0}=1, \quad T_{1}=\xi, \quad U_{0}=0, \quad U_{1}=1, \\
N\left(\xi, \omega_{n}\right)=\gamma\left(\xi, \omega_{n}\right)+j \delta\left(\xi, \omega_{n}\right),
\end{gathered}
$$

where $\gamma\left(\xi, \omega_{n}\right)$ and $\delta\left(\xi, \omega_{n}\right)$ represent the real and imaginary parts of the polynomial $N\left(\xi, \omega_{n}\right)$, and they are determined based on the following equations:

$$
\begin{aligned}
& \gamma\left(\xi, \omega_{n}\right)=\sum_{k=0}^{m} b_{k}(-1)^{k} \omega_{n}^{k} T_{k}(\xi), \\
& \delta\left(\xi, \omega_{n}\right)=\sqrt{1-\xi^{2}} \sum_{k=0}^{m} b_{k}(-1)^{k+1} \omega_{n}^{k} U_{k}(\xi) .
\end{aligned}
$$

By connecting (13) with (14) and (18), it is obtained that

$$
\begin{gathered}
\alpha\left(\xi, \omega_{n}\right)+j \beta\left(\xi, \omega_{n}\right)+\left[K_{p}\left(-\xi \omega_{n}+j \omega_{n} \sqrt{1-\xi^{2}}\right)+K_{i}\right] \\
\cdot\left[\gamma\left(\xi, \omega_{n}\right)+j \delta\left(\xi, \omega_{n}\right)\right]=0 .
\end{gathered}
$$

By separating the real and imaginary parts from (20) and by solving it, the following system of equations is obtained:

$$
\begin{aligned}
K_{p} & {\left[\xi \cdot \omega_{n} \cdot \gamma\left(\xi, \omega_{n}\right)+\sqrt{1-\xi^{2}} \cdot \omega_{n} \cdot \delta\left(\xi, \omega_{n}\right)\right] } \\
& -K_{i} \cdot \gamma\left(\xi, \omega_{n}\right)=\alpha\left(\xi, \omega_{n}\right), \\
K_{p} & {\left[\xi \cdot \omega_{n} \cdot \delta\left(\xi, \omega_{n}\right)-\sqrt{1-\xi^{2}} \cdot \omega_{n} \cdot \gamma\left(\xi, \omega_{n}\right)\right] } \\
& -K_{i} \cdot \delta\left(\xi, \omega_{n}\right)=\beta\left(\xi, \omega_{n}\right) .
\end{aligned}
$$


By solving the system of equations at $\omega_{n} \neq 0,0 \leq \xi<1$ the expressions for the parameters $K_{p}$ and $K_{i}$ of the PI controller are obtained as follows:

$$
\begin{gathered}
K_{p}=\frac{\alpha\left(\xi, \omega_{n}\right) \cdot \delta\left(\xi, \omega_{n}\right)-\beta\left(\xi, \omega_{n}\right) \cdot \gamma\left(\xi, \omega_{n}\right)}{\omega_{n} \cdot \sqrt{1-\xi^{2}} \cdot\left[\gamma^{2}\left(\xi, \omega_{n}\right)+\delta^{2}\left(\xi, \omega_{n}\right)\right]} \\
K_{i}=-\frac{\xi \cdot\left(\beta\left(\xi, \omega_{n}\right) \cdot \gamma\left(\xi, \omega_{n}\right)-\alpha\left(\xi, \omega_{n}\right) \cdot \delta\left(\xi, \omega_{n}\right)\right)}{\sqrt{1-\xi^{2}} \cdot\left[\gamma^{2}\left(\xi, \omega_{n}\right)+\delta^{2}\left(\xi, \omega_{n}\right)\right]} \\
+\frac{\sqrt{1-\xi^{2}} \cdot\left(\beta\left(\xi, \omega_{n}\right) \cdot \delta\left(\xi, \omega_{n}\right)+\alpha\left(\xi, \omega_{n}\right) \cdot \gamma\left(\xi, \omega_{n}\right)\right)}{\sqrt{1-\xi^{2}} \cdot\left[\gamma^{2}\left(\xi, \omega_{n}\right)+\delta^{2}\left(\xi, \omega_{n}\right)\right]}, \\
K_{i}=\omega_{n}\left[\xi+\sqrt{1-\xi^{2}}\right. \\
\left.\quad \frac{\beta\left(\xi, \omega_{n}\right) \cdot \delta\left(\xi, \omega_{n}\right)+\alpha\left(\xi, \omega_{n}\right) \cdot \gamma\left(\xi, \omega_{n}\right)}{\beta\left(\xi, \omega_{n}\right) \cdot \gamma\left(\xi, \omega_{n}\right)-\alpha\left(\xi, \omega_{n}\right) \cdot \delta\left(\xi, \omega_{n}\right)}\right] \cdot K_{p} .
\end{gathered}
$$

For $\omega_{n}=0$, the system of (21) gives the solution:

$$
K_{i}=0 .
$$

Equation (25) represents a singular straight line which, together with the curve described by (22), represents the closed contour of the region of possible solutions, in the plane of variable parameters $K_{p}, K_{i}$ shown in Figure 3.

For $\xi=1$, the system of (21) is reduced to one equation described by

$$
K_{i}=\omega_{n} K_{p}-\frac{\alpha\left(1, \omega_{n}\right)}{\gamma\left(1, \omega_{n}\right)} .
$$

Equations (22) through (26) completely define the parameter $\left(K_{p}, K_{i}\right)$ plane for the selected value of the damping coefficient $\xi=$ const., $0 \leq \xi \leq 1$, at the change of undamped frequency $\omega_{n}=\left(0, \omega_{n \max }\right)$. The parameter plane with its stability limits is presented in Figure 3.

From Figure 3, three characteristic areas particularly stand out for the following:

(i) $\xi=0$, the system is at the stability limit and has oscillatory character, the area below the curve $\xi=0$ is the stability area, and the area above the curve is the nonstability area;

(ii) $\xi=1$, the system has critical damping, and the area below the curve $\xi=1$ is the area in which the system has monotonous (aperiodic) character. The critically damped response is desired in a lot of applications because it represents the fastest aperiodic response possible;

(iii) $\xi=$ const., the curve for the required degree of system damping from which the variable parameters of the controller $\left(K_{p}, K_{i}\right)$ can be chosen.

Figure 3 allows reading the maximum value of integral gain at the point $A, K_{i}=K_{i \max }$, and the corresponding proportional

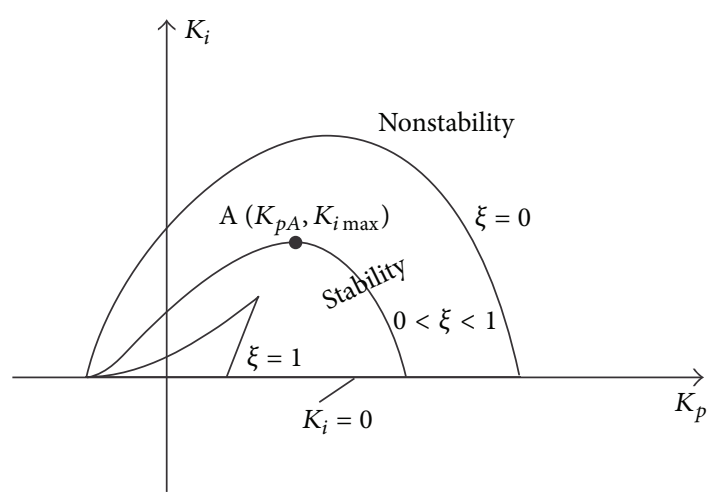

FIgURE 3: Parameter plane with the stability limit.

gain $K_{p A}$. Selection of the maximum integral gain will not always yield good results regarding robustness defined by phase margin $\left(\varphi_{m}\right)$, gain margin $\left(g_{m}\right)$, and robustness measure $s_{m}$ [27]. This problem is particularly noticeable in systems of high order. In the character of response of the closed loop, it is reflected in the increased overshoot $(O)$ and the increased settling time $t_{s}$.

In order to solve this problem, the area of selection of parameters of the controller $\left(K_{p}, K_{i}\right)$ has been narrowed by taking out the area in which the system will possess good settling time $\left(t_{s}\right)$ on the corresponding curve $\xi=$ const.

In order to have a system with good settling time, it is necessary that all real parts of the poles of the transfer function of the closed loop should have a location to the left of the straight line $\sigma_{m}=$ const. (from Figure 2). The area from the " $s$ " plane to the left of the straight line $\sigma_{m}=$ const. (Figure 2) is thus mapped in the area of the corresponding settling time $\sigma_{\min }=$ const., in the parameter plane of tuning parameters of the controller $\left(K_{p}, K_{i}\right)$ as follows:

$$
s=-\sigma+j \omega
$$

Based on (12) and (27), it is clear that

$$
\sigma=\xi \cdot \omega_{n}, \quad \omega=\omega_{n} \sqrt{1-\xi^{2}} .
$$

Here it is necessary to find the minimum value of undamped frequency that will satisfy the condition:

$$
\left|\sigma_{\min }\right| \geq\left|\sigma_{m}\right|, \quad\left|\xi \cdot \omega_{n \min }\right| \geq\left|\sigma_{m}\right| .
$$

In order to have all real parts of the complex number $s=$ $-\sigma$ to the left of the straight line $\sigma_{m}$, it is necessary to find the variable parameters $\left(K_{p}, K_{i}\right)$ that will satisfy those conditions. This is realized by replacing the complex number $s=-\sigma_{\min }$ in (9), so that the characteristic equation now obtains the form:

$$
K_{i \min }=\sigma_{\min } \cdot K_{p}-\frac{f_{1}\left(-\sigma_{\min }\right)}{N\left(-\sigma_{\min }\right)}, \quad \sigma_{\min }=\xi \cdot \omega_{n \min } .
$$

The value of undamped frequencies $\omega_{n \min }$ and $\omega_{n \text { max }}$, whose location is identified in Figure 2, is read from the graphs $K_{p}\left(\xi, \omega_{n}\right)=0$ and $K_{i}\left(\xi, \omega_{n}\right)=0$, as it is shown in Figure 4 . 


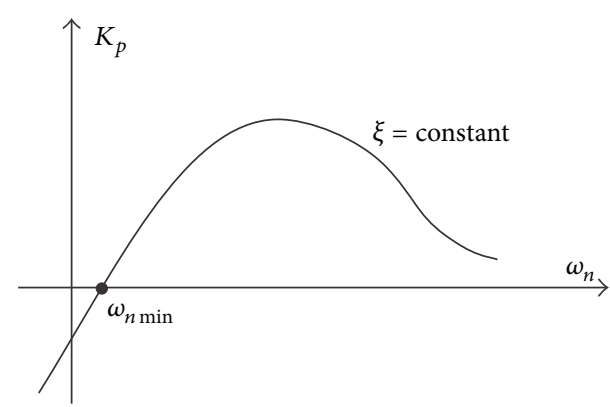

(a)

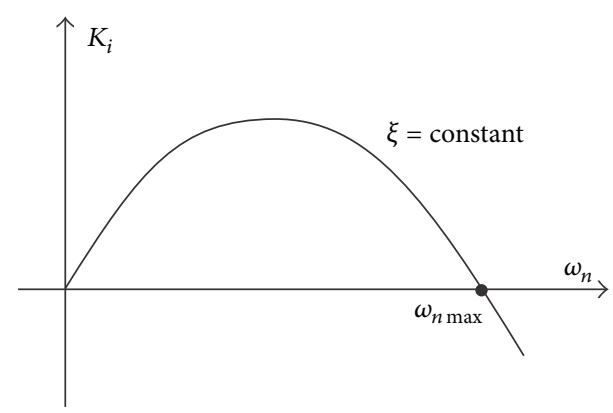

(b)

FIGURE 4: Change of the proportional and integral gains of the controller as a function of undamped frequency for the required damping coefficient.

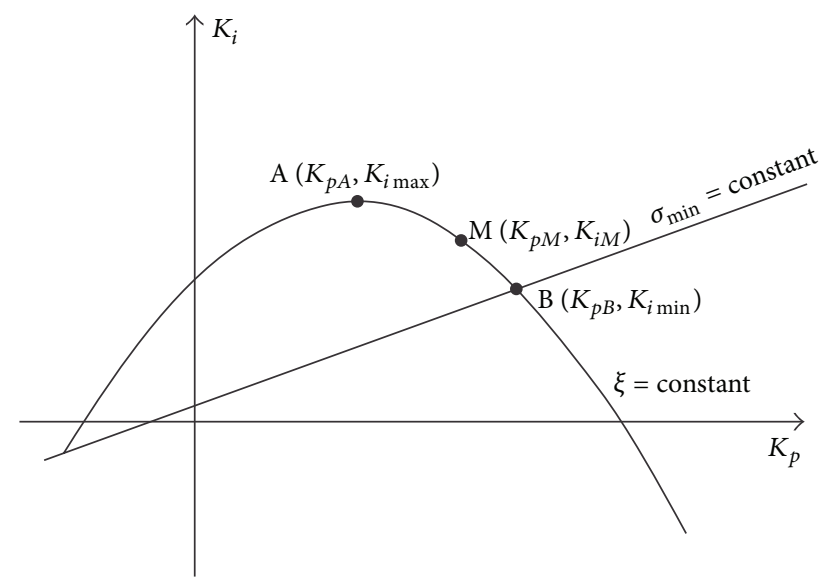

Figure 5: Parameter plane with the boundaries of maximum and minimum integral gains for the required damping degree of the closed loop.

The graphical interpretation of (30), $\sigma_{\min }=$ const., in the parameter plane $\left(K_{p}, K_{i}\right)$ of the required damping coefficient $\xi=$ const., is shown in Figure 5.

The straight line $\sigma_{\min }=$ const. intersects the curve of the required damping coefficient $\xi=$ const. in the parameter plane at the point $B$ with the coordinates $B\left(K_{p B}, K_{i \text { min }}\right)$. At the point $B$, the integral gain has a minimum value. The point $M$ with the desired coordinates $M\left(K_{p M}, K_{i M}\right)$ lies at the part of the curve $\xi=$ const. between the points $A$ and $B$, at the interval of undamped frequencies $\omega_{n}=\left(\omega_{n \text { min }}, \omega_{n \text { max }}\right)$. The lower the order of the process, the closer the point $M$ to the point $A\left(K_{p A}, K_{i \max }\right)$, and the higher the order of the process, the closer the point $M$ to the point $B\left(K_{p B}, K_{i \text { min }}\right)$. In this narrow range, by selecting the appropriate step, it is very easy to find the point $M$ with the parameters of the controller that will guarantee quality operation of the automatic control system.

\section{Control of a Process with a Long Transmission Line}

In order to show the efficiency of the proposed method of design of the PI controller, simulation in the program package
MATLAB for the transfer function of the process $W_{P 1}(s)$ has been carried out as follows:

$$
\begin{aligned}
W_{P 1}(s)=1 \times( & 5.2 \cdot 10^{-25} s^{10}+9.23 \cdot 10^{-22} s^{9} \\
& +9.677 \cdot 10^{-19} s^{8}+7.838 \cdot 10^{-16} s^{7} \\
& +4.592 \cdot 10^{-13} s^{6}+2.072 \cdot 10^{-10} s^{5}+\cdots \\
& +7.257 \cdot 10^{-8} s^{4}+1.755 \cdot 10^{-5} s^{3} \\
& \left.+2.962 \cdot 10^{-3} s^{2}+0.243 s+1.248\right)^{-1} .
\end{aligned}
$$

The transfer function described by (31) represents a mathematical model of a real process used in industry. It is a pump controlled hydromotor, where the variable flow pump and the hydromotor of constant flow are connected by means of a long transmission line. The complete identification of this transfer function was done in paper [13]. The motive for taking exactly this transfer function of the process for testing the proposed method is its high order-it is of the tenth order. When the system is of high order, as in the case of the transfer function $W_{P 1}(s)$, then the poles which are closest to the imaginary axis in the complex plane have the decisive influence on the character of the transition process. The proposed methodology allows location of such poles of the transfer function at a safe distance from the imaginary axis; that is, it allows the closed loop of the system to have all its poles to the left of the straight line $\sigma_{m}$, which is presented in Figure 2.

Based on the programme created in MATLAB and according to the procedure proposed in the paper, the parameters of the PI controller can be determined for any transfer function of the process described by (4), so that the closed loop of the system could possess the required damping coefficient and good settling time.

The flow of the programme written in MATLAB is represented by the algorithm shown in Figure 6.

The result of operation of the programme is the graphical interpretation of parameters of the controller shown in Figure 7, for different values of the damping coefficient $0 \leq$ $\xi \leq 1$ of the closed loop of the automatic control system. The stability limit is marked by the curve $\xi=0$, from which it is possible to read the value of the critical proportional gain 


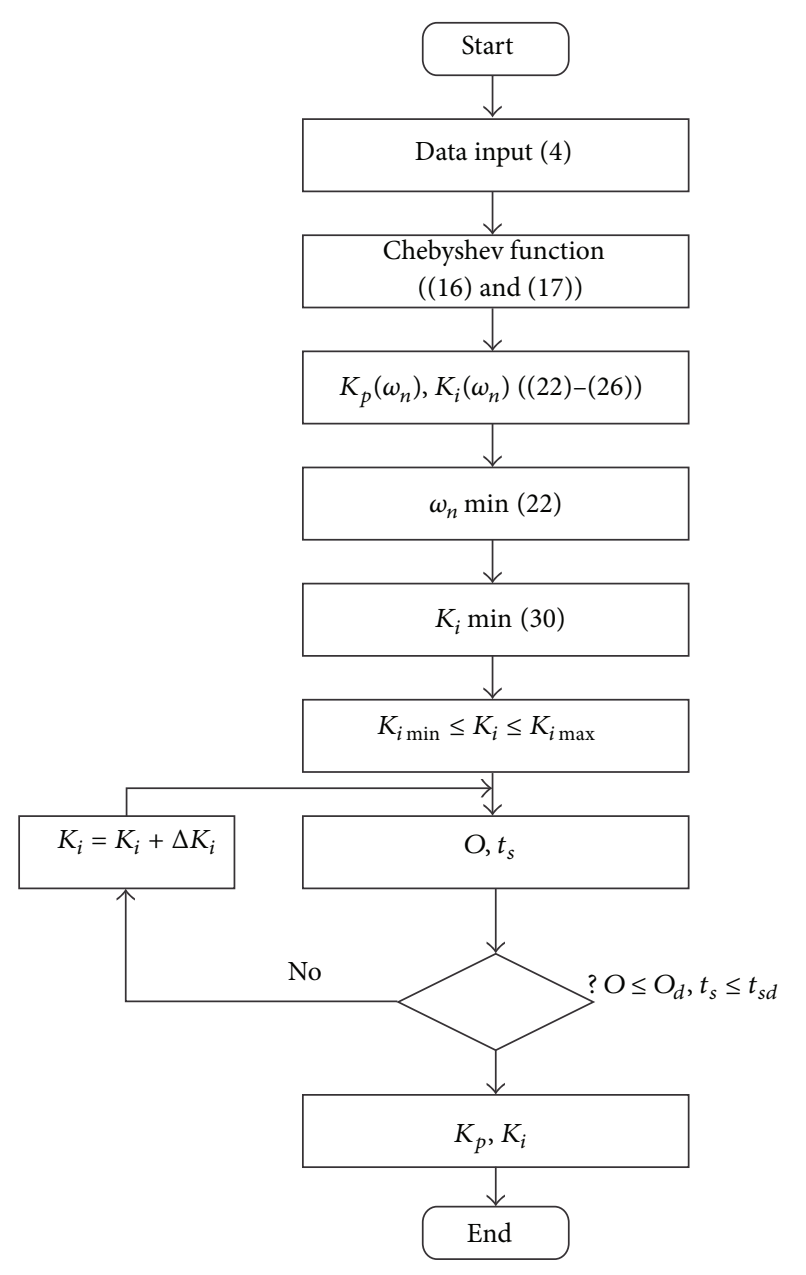

FIgURE 6: Algorithm for determination of parameters of the PI controller.

$K_{p k r}=30.39$. The values of the maximum integral gain $K_{i \max }$ and the corresponding proportional gain $K_{p A}$ for different values of the damping coefficient $\xi$ are read from Figure 7. The values of parameters of the controller obtained from Figure 7 are shown in Table 1.

The responses of the automatic control system presented in Figure 8 are recorded for the designed values of parameters of the PI controller from Table 1 as well as for the critical value of the proportional gain $K_{p k r}=30.39$ at $\xi=0$. From Figure 8 it can be seen that high overshoots and high values of settling time are obtained in the step response, even for higher values of the damping coefficient $(\xi=0.6,0.8$, and 1.0) of the closed loop. The values of overshoot and settling time are shown in Table 1.

Figure 9 shows how the designed controller, in accordance with the minimum of the IE criterion, rejects the load caused by the action of disturbance $D=1$ at the value of reference $X_{r}=0$. From Figure 9 it is possible to conclude that good results from the aspect of load disturbance rejection can be expected for the values of the damping coefficient $\xi \geq 0.4$. Figure 9 also shows that in an uncontrolled process the load of the system which is caused by disturbance will not

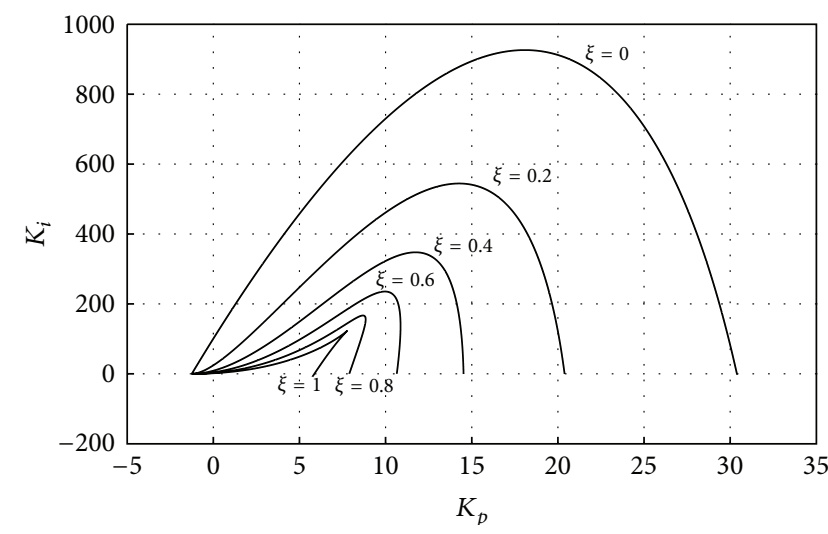

FIGURE 7: Parameter plane for different values of the damping coefficient with the stability limits.

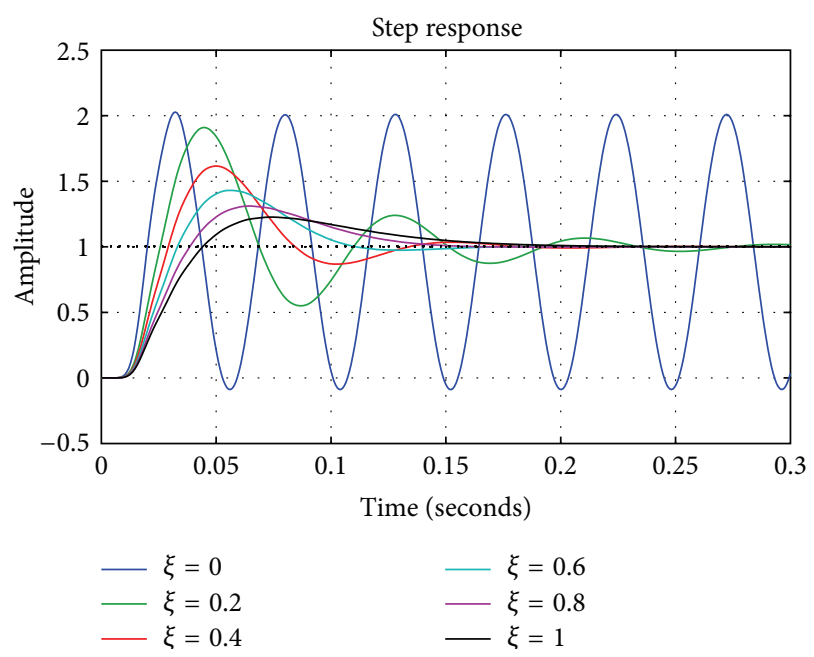

FIGURE 8: Response of the system for the reference value $X_{r}=1$ at $K_{i \text { max }}$, for changes of the damping coefficient $0 \leq \xi \leq 1$.

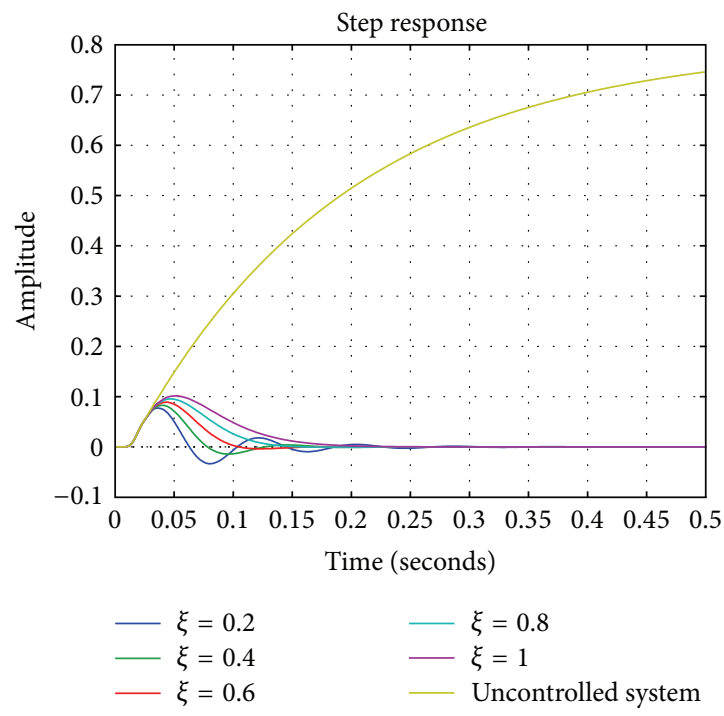

FIGURE 9: Response of the system to the action of disturbance $D=1$ at $K_{i \max }$, for changes of the damping coefficient $0.2 \leq \xi \leq 1$. 


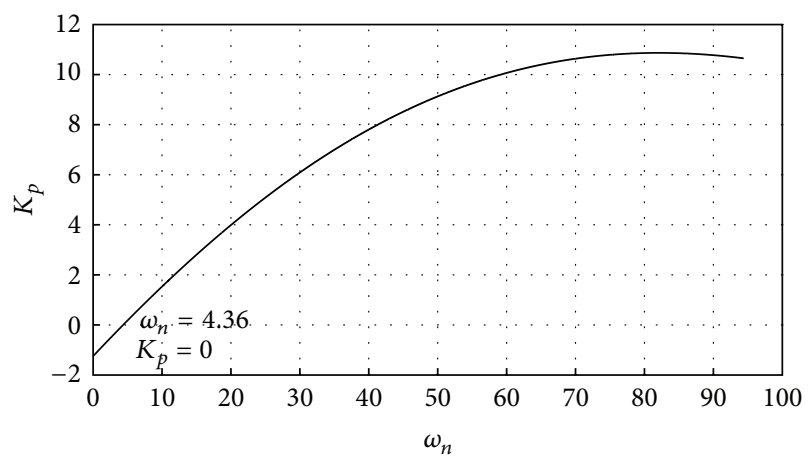

(a)

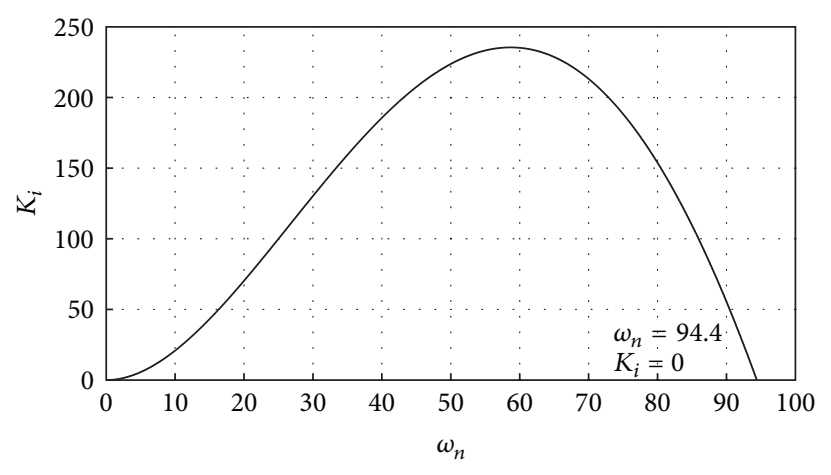

(b)

FIGURE 10: Boundary values of the undamped frequency $\omega_{n \min }$ and $\omega_{n \max }$ for the damping coefficient of the closed loop $\xi=0.6$.

TABLE 1: Results of design of the PI controller with performances.

\begin{tabular}{lcccccc}
\hline Damping $\xi$ & $K_{p A}$ & $K_{i \max }$ & Overshoot $(\%)$ & Settling time $(\mathrm{ms})$ & Phase margin $\varphi_{m}($ degrees $)$ & Gain margin $g_{m}$ \\
\hline 0.2 & 14.38 & 544.4 & 91 & 265 & 15.8 & 27.2 \\
0.4 & 11.83 & 347.6 & 61 & 169 & 35.9 & 2.1 \\
0.6 & 10.02 & 235.4 & 43 & 143 & 42.8 & 2.6 \\
0.8 & 8.665 & 166.9 & 31 & 140 & 48.8 & 3.1 \\
1.0 & 7.77 & 122.9 & 23 & 178 & & 3.57 \\
\hline
\end{tabular}

be rejected. However, according to this criterion, satisfactory values of overshoot, settling time, and stability margin will not be obtained, which is best seen from Table 1 .

In order to eliminate the undesired values of increased overshoot and settling time, for the chosen damping coefficient $\xi=0.6$, the settling time has been estimated according to $\sigma_{\min }=\xi \omega_{n \min }$. The values of the minimum undamped frequency $\omega_{n \text { min }}$ and $\omega_{n \max }$ are read from Figure 10.

The minimum value of the integral gain $K_{i \text { min }}=29.75$ and the corresponding proportional gain $K_{p}=10.73$ is thus obtained in the parameter plane, as it can be seen from Figure 11. Going along the part of the curve shown in Figure 11, from the point of minimum integral gain toward the point of maximum integral gain, with the appropriate step (automatically generated by the program) and recording the response, it is very easy to reach the point on the curve with the parameters of the controller that will give best performances for the given process.

Figure 12 shows the step response of the closed loop with the parameters of the controller read from Figure 11. It can be seen from Figure 12 that for the designed values of parameters of the controller $K_{p}=10.78$ and $K_{i}=57.15$, the closed loop of the automatic control system possesses good performances (the overshoot of $9.98 \%$ and the settling time of $72.8 \mathrm{~ms}$ ).

\section{Comparison with Other Methods}

The PI controller designed by means of the methodology presented in this paper for the process described by (31) has been compared with the Ziegler-Nichols (ZN) [1] and TyreusLuyben (TL) [28] methods for tuning of parameters of the PI controller. Those are two very frequent methods of tuning

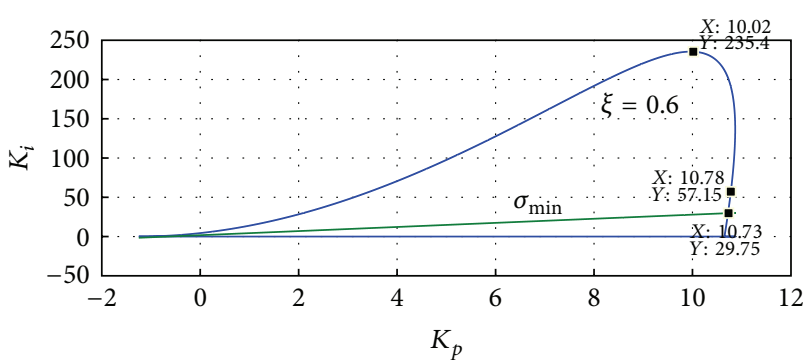

Figure 11: Parameter plane for the required damping degree and required settling time.

of parameters of controllers in industrial practice. The results of comparison in the step response for the described process are shown in Figure 13. The complete performances from the aspect of response, relative stability and robustness are presented in Table 2.

Based on the results presented in Table 2, it can be seen that the Ziegler-Nichols method gives a very oscillatory response characterized by the overshoot of $62.3 \%$ with the settling time of $148 \mathrm{~ms}$ and very bad robustness (phase margin $28^{\circ}$, gain margin 1.89 , and stability margin 0.48 ). The Tyreus-Luyben method gives a well-damped response characterized by the overshoot of $13.6 \%$, but with a very high value of settling time of $195 \mathrm{~ms}$. From the aspect of robustness, the Tyreus-Luyben method gives good results. The method proposed in this paper gives a well-damped response characterized by the overshoot of $9.98 \%$ with considerably better settling time of $72 \mathrm{~ms}$ in comparison with the $\mathrm{ZN}$ and TL methods. From the aspect of robustness, the 
TABLE 2: Comparative presentation of the results of design of the PI controller with performances for three methods.

\begin{tabular}{lccccccc}
\hline Method & $K_{p}$ & $K_{i}$ & Overshoot (\%) & Settling time $t_{s}(\mathrm{~ms})$ & $\begin{array}{c}\text { Phase margin } \\
\varphi_{m}(\text { degrees })\end{array}$ & Gain margin $g_{m}$ & Stability margin $s_{m}$ \\
\hline $\begin{array}{l}\text { Ziegler-Nichols } \\
\text { (ZN) }\end{array}$ & 13.6775 & 341.8875 & 62.3 & 148 & 28 & 1.89 & 0.48 \\
$\begin{array}{l}\text { Tyreus-Luyben } \\
\text { (TL) }\end{array}$ & 9.4379 & 89.3741 & 13.6 & 195 & 54.6 & 3.05 & 0.62 \\
$\begin{array}{l}\text { Proposed method } \\
(\mathrm{PM})\end{array}$ & 10.78 & 57.15 & 9.98 & 72 & 56.5 & 2.74 & 0.64 \\
\hline
\end{tabular}

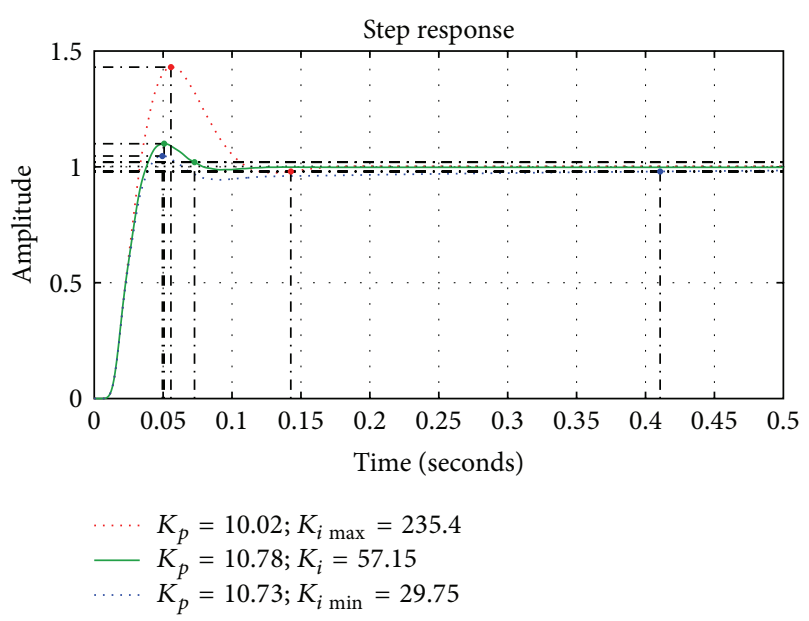

FIGURE 12: System response at the defined damping coefficient $\xi=$ 0.6 and the required settling time.

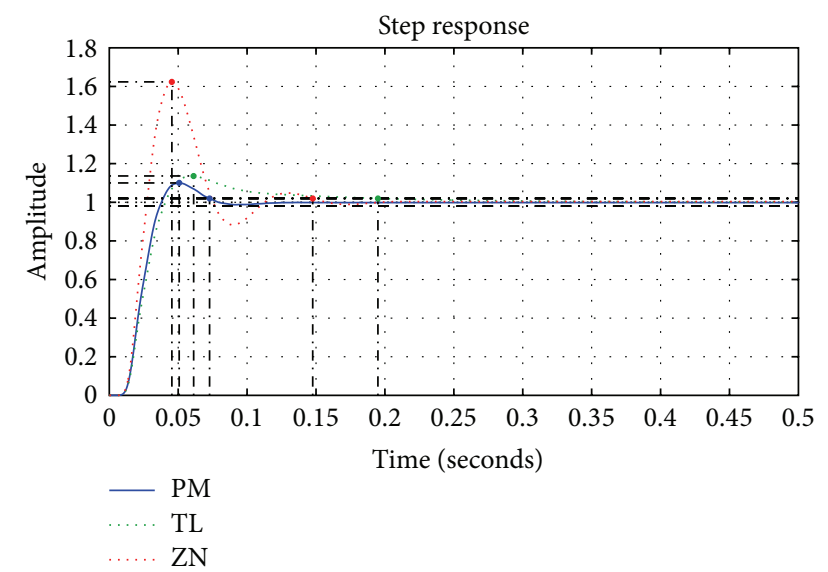

FIGURE 13: Comparative presentation of the system responses, to the action of reference $X_{r}=1$, for the proposed method (PM), the Ziegler-Nichols (ZN) method, and the Tyreus-Luyben (TL) method.

proposed method also gives a better result (phase margin $56.5^{\circ}$, gain margin 2.74 , and stability margin 0.64 ) too.

The results of comparison from the aspect of load disturbance rejection are presented in Figure 14. From Figure 14 it is possible to see that the proposed method for the values of the damping coefficient $(\xi=0.5$ and $\xi=0.6$ ) quite satisfactorily rejects the load caused by the action of disturbance $D=1$

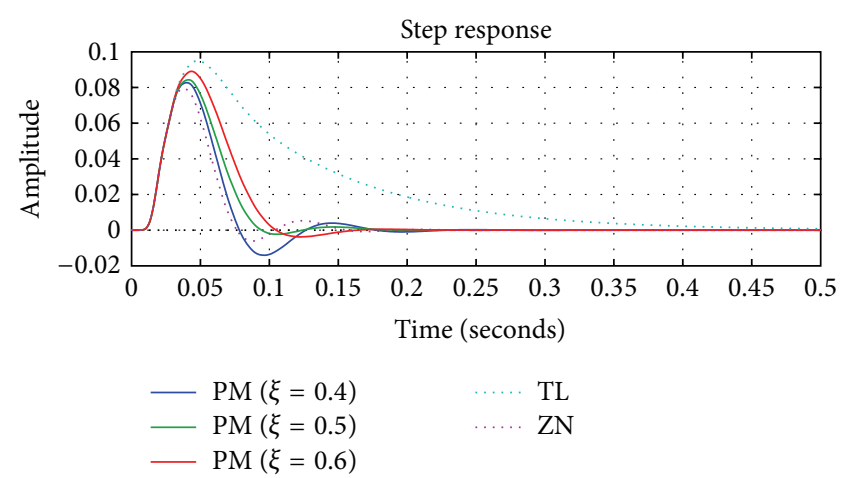

FIGURE 14: Comparative presentation of the system response to the action of load disturbance $D=1$, for the proposed method (PM), the Ziegler-Nichols method (ZN) and the Tyreus-Luyben method (TL).

in comparison with the Ziegler-Nichols method and does it considerably better in relation to the Tyreus-Luyben method.

Remark. It is well known that the Ziegler-Nichols frequency method cannot be applied to the processes of the first and second orders because such processes cannot be brought to the oscillatory stability limit by changing the proportional gain. Also, the Ziegler-Nichols method and some other similar methods cannot be applied to unstable processes. The proposed methodology does not have any restrictions referring to the order of the system. It can be very efficiently applied to low-order processes. Another advantage of this method is that it can also be applied to unstable processes of low order.

\section{Conclusion}

This paper has developed an efficient and simple graphical methods for design of the PI controller, which achieves high performances for a broad range of linear processes. For design, it is necessary to know the transfer functions of the process. The processes of high order have been considered in the paper. In comparison with the procedures for tuning of the PI controller proposed in literature, the method described in this paper is characterized by great simplicity and clear engineering specifications. The results of simulations show good robustness in relation to unmodelled dynamics as well as superiority over some other methods of tuning of controllers. The proposed method is suitable for on line 
real-time implementation and for auto tuning of the PI controller. The methodology of design of the PI controller presented in this paper is simple and can easily be adopted by industry. The extension of this method to design of the PID controller is underway.

\section{List of Symbols}

$\begin{array}{ll}a_{k}, b_{k}: & \text { Coefficients of the transfer function } \\ D: & \text { Load disturbance } \\ e(t): & \text { Control value error } \\ f(s): & \text { Characteristic polynomial } \\ f_{1}(s): & \text { Part of the characteristic polynomial } f(s) \\ g_{m}: & \text { Gain margin } \\ \mathrm{IE}: & \text { Integrated error } \\ K_{i}: & \text { Integral gain of the controller } \\ K_{p}: & \text { Proportional gain of the controller } \\ N(s), M(s): & \text { Polynomial of the numerator and } \\ & \text { denominator of the transfer function } \\ O(\%): & \text { Overshoot } \\ s: & \text { Complex number } \\ s_{m}: & \text { Stability margin } \\ T_{k}(\xi), U_{k}(\xi): & \text { Chebyshev functions of the first and } \\ t(s): & \text { second kinds } \\ t_{s}(s): & \text { Time } \\ U(s): & \text { Settling time } \\ W_{P}, W_{R}: & \text { Control signal } \\ X(s): & \text { Transfer functions of the process and the } \\ X_{r}(s): & \text { controller }\end{array}$

Greek Letters
[3] D. B. Ender, "Process control performance: not as good as you think," Control Engineering, vol. 40, pp. 180-186, 1993.

[4] W. L. Bialkowski, "Dreams and reality: a view from both sides of the gap," Pulp Paper Canada, vol. 94, pp. 19-25, 1993.

[5] M. A. Hersh and M. A. Johnson, "A study of advanced control systems in the workplace," Control Engineering Practice, vol. 5, no. 6, pp. 771-778, 1997.

[6] S. Yamamoto and I. Hashimoto, "Present status and future needs: the view from Japanese industry," in Proceedings of the International Conference on Chemical Process Control Chemical Process Control, 1991.

[7] K. J. Astrom and T. Hagglund, PID Controllers: Theory, Design, and Tuning, Instrument Society of America, Research Triangle Park, NC, USA, 2nd edition, 1995.

[8] F. G. Shinskey, "How good are our controllers in absolute performance and robustness?" Measurement and Control, vol. 23, no. 4, pp. 114-121, 1990.

[9] K. J. Astrom and T. Hagglund, "New tuning methods for PID controllers," in Proceedings of the European Control Conference, pp. 2456-2462, Rome, Italy, 1995.

[10] K. J. Åström, H. Panagopoulos, and T. Hägglund, "Design of PI controllers based on non-convex optimization," Automatica, vol. 34, no. 5, pp. 585-601, 1998.

[11] Y. G. Wang and H. H. Shao, "Optimal tuning for PI controller," Automatica, vol. 36, no. 1, pp. 147-152, 2000.

[12] M. R. Mataušek and T. B. Šekara, "PID controller frequencydomain tuning for stable, integrating and unstable processes, including dead-time," Journal of Process Control, vol. 21, no. 1, pp. 17-27, 2011.

[13] N. Nedić, L. J. Dubonjić, and V. Filipović, "Design of constant gain controllers for the hydraulic control system with a long transmission line," Forschung im Ingenieurwesen, vol. 75, no. 4, pp. 231-242, 2011.

[14] Yu. I. Neimark, "The structure of the D-decomposition of a space of polynomials and the diagrams of Vyšnegradskiì and Nyquist," Doklady Akademii Nauk SSSR, vol. 59, pp. 853-856, 1948 (Russian).

[15] Y. I. Neimark, "Search for the parameter values that make automatic control system stable," Automatika i Telemehanika, vol. 9, no. 3, pp. 190-203, 1948.

[16] D. Mitrovic, "Graphical analysis and synthesis of feedback control systems. I-theory and analysis, II-synthesis, III-sampleddata feedback control systems," AIEE Transactions, vol. 77, pp. 476-496, 1959.

[17] D. Siljak, "Analysis and synthesis of feedback control systems in the parameter plane. I-linear continuous systems, II-sampleddata systems," AIEE Transactions, vol. 83, pp. 449-466, 1964.

[18] D. Siljak, "Generalization of the parameter plane method," IEEE Transactions on Automatic Control, vol. 11, no. 1, pp. 63-70, 1966.

[19] D. Siljak, Nonlinear Systems: The Parameter Analysis and Design, Wiley, New York, NY, USA, 1969.

[20] E. N. Gryazina and B. T. Polyak, "Stability regions in the parameter space: D-decomposition revisited," Automatica, vol. 42, no. 1, pp. 13-26, 2006.

[21] K. J. Astrom and B. Wittenmark, Computer Controlled Systems. Theory and Design, Prentice-Hall, 1997.

[22] K. Zhou, J. C. Doyle, and K. Glover, Robust and Optimal Control, Prentice-Hall, 1996.

[23] A. E. Barabanov, Design of Minimax Controllers, Sankt Peterburg University, 1996. 
[24] S. P. Bhattacharyya, H. Chapellat, and L. H. Keel, Robust Control: The Parametric Approach, Prentice-Hall, 1995.

[25] M. T. Ho, A. Datta, and S. P. Bhattacharyya, "Control system design using low order controllers: constant gain, PI and PID," in Proceedings of the American Control Conference, pp. 571-578, Albuquerque, NM, USA, June 1997.

[26] A. Datta, S. P. Bhattacharyya, and L. H. Keel, Linear Control Theory. Structure, Robustness, and Optimization, CRC Press, Boca Raton, Fla, USA, 2009.

[27] K. J. Åström and R. M. Murray, Feedback Systems. An Introduction for Scientists and Engineers, Princeton University Press, Princeton, NJ, USA, 2008.

[28] B. D. Tyreus and W. L. Luyben, "Tuning PI controllers for integrator/dead time processes," Industrial and Engineering Chemistry Research, vol. 31, no. 11, pp. 2625-2628, 1992. 


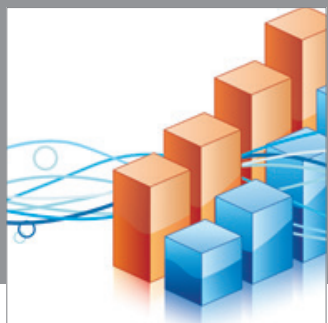

Advances in

Operations Research

mansans

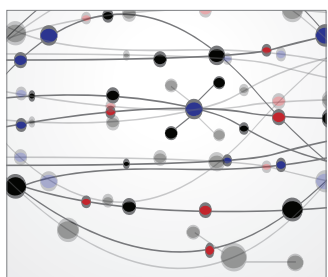

The Scientific World Journal
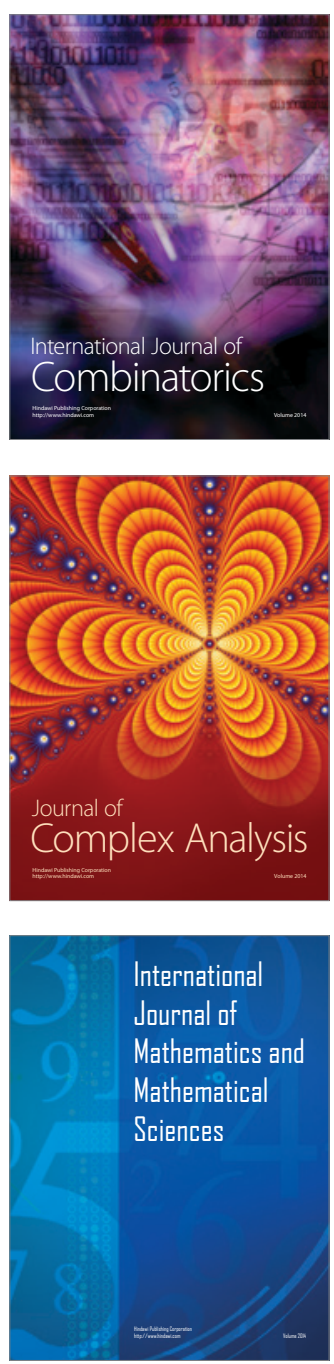
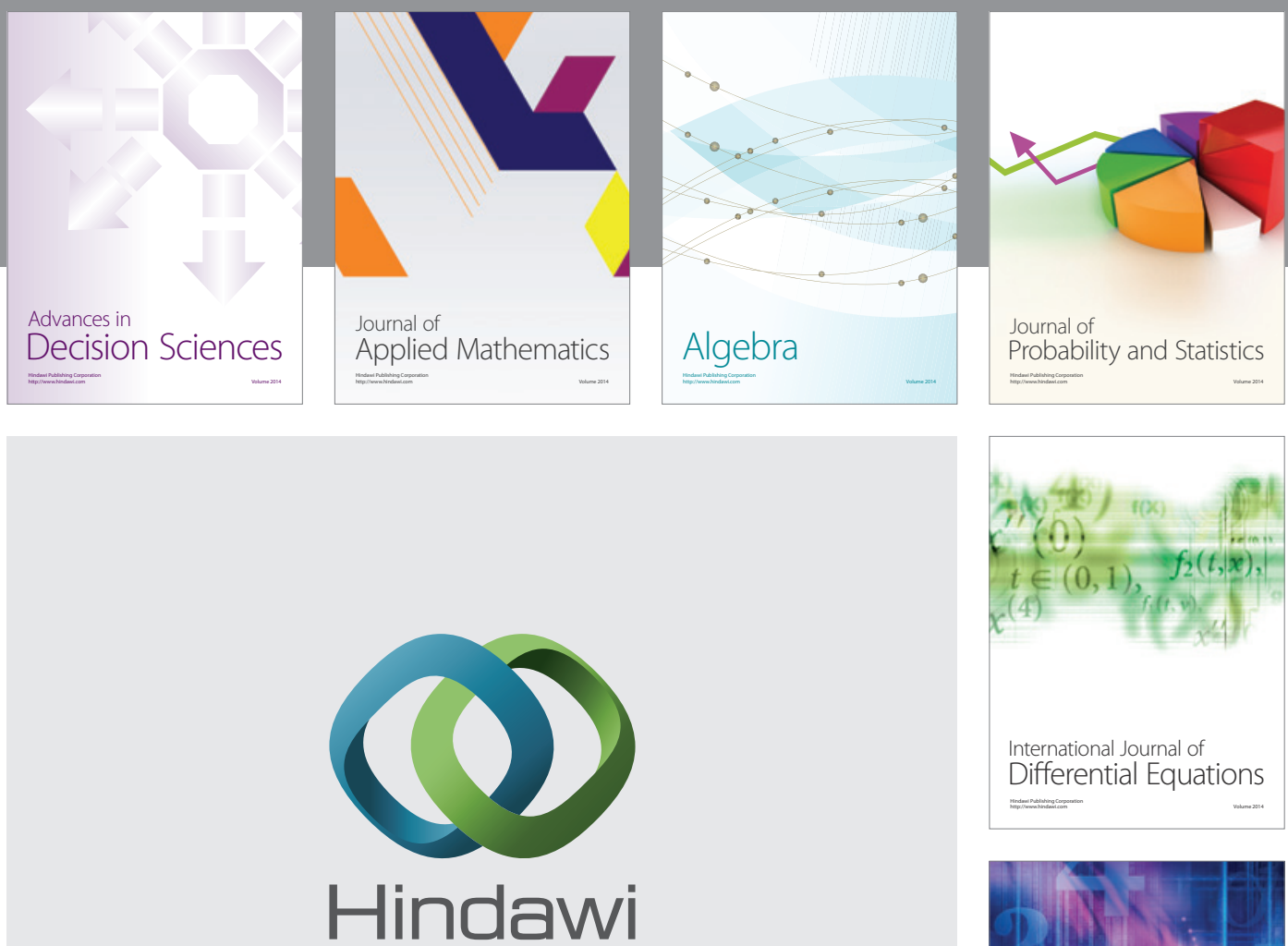

Submit your manuscripts at http://www.hindawi.com
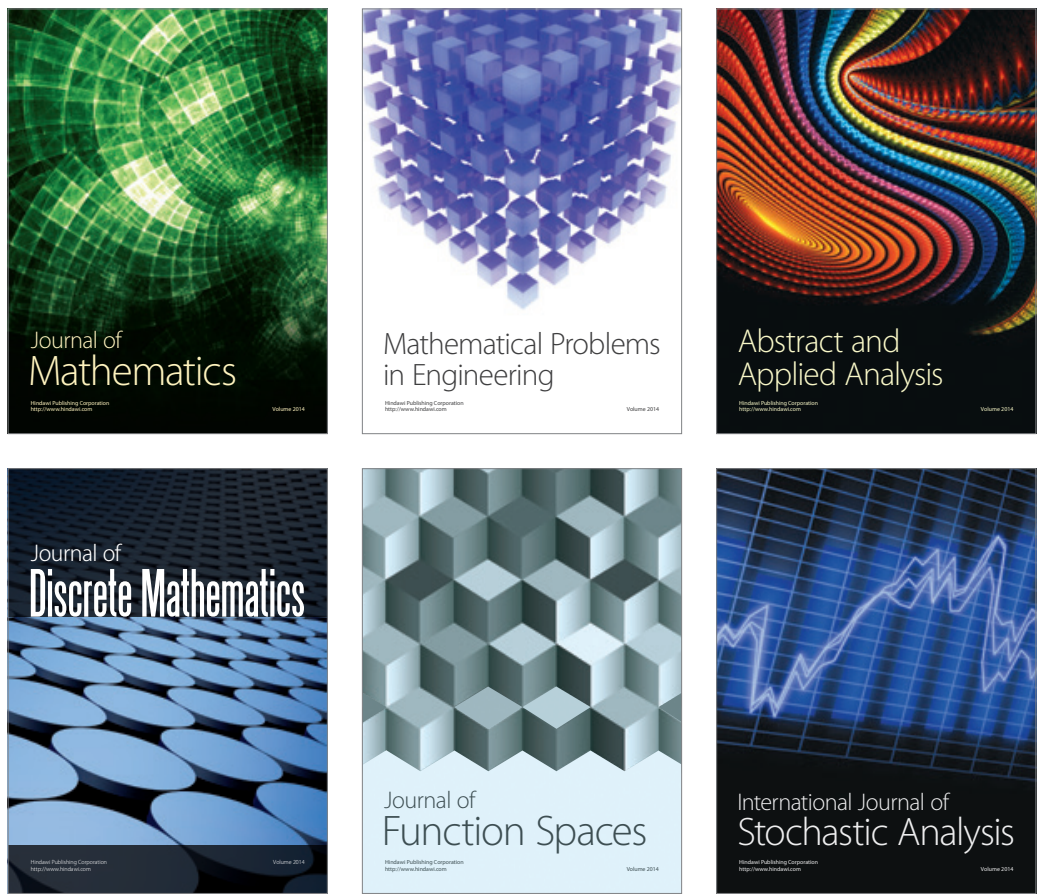

Journal of

Function Spaces

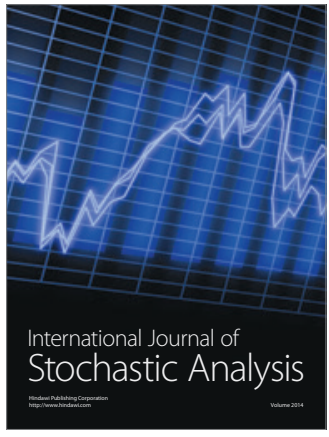

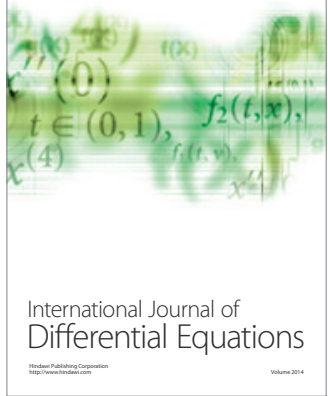
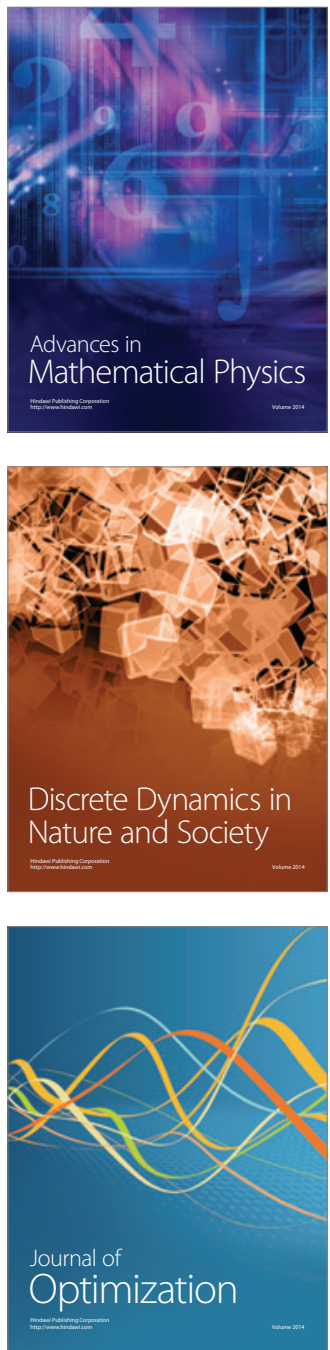